\title{
COLLECTOR'S CURVES \& THE PREDICTION OF STEADY STATES IN SPECIES DIVERSITY
}

\author{
ROSENZWEIG, Michael L., Dept. Ecology \& Evolutionary Biology, \\ U. of Arizona, Tucson, AZ 85721, USA; SCARAB@CCIT.ARIZONA.EDU
}

The basic theory: The average species' geographical range declines as diversity grows. Smaller ranges lead to lower speciation rates (per species) because species with smaller ranges generally experience fewer isolating barriers and can evolve fewer new gene loci. Smaller ranges should also lead to higher extinction rates (per species). A consequence of these correlations should be that species diversity tends toward a steady state.

This does not mean that life should have reached and maintained a single steady state throughout the Phanerozoic but that it should have tracked a series of steady states. It also implies that Willis got it backwards when he tried to explain the correlation between species' ages and the geographical areas they cover: he should have said that species with larger areas tend to live longer, not that they tend to expand their areas as they age.

Much paleobiological evidence suggests that life has indeed tracked its steady states. None suggests the alternative, i.e. that diversity has been increasing for hundreds of millions of years, always far from any theoretical steady state.

Interprovincial species-area curves also support the relevance of steady state theory to diversity. This new type of collector's curve shows that evolution maintains more species in larger biogeographical provinces. But the logarithmic slopes of interprovincial curves (i.e., their $z$-values) tend toward 1.00, suggesting that variation in the number of biogeographical provinces has had little to do with variation in the world's species diversity during the Phanerozoic.

Another new type of collector's curve - the number of new species that accumulate with time - also seems to have a logarithmic slope near 1.00. This suggests that another diversity statistic - the rate of species accumulation among marine invertebrates - has remained nearly steady during the Phanerozoic (except during periods of recovery from mass extinction). Yet there is some weak evidence that holds out the chance that it declined by about $50 \%$. Careful, pinpoint collection of fossils from key strata would resolve this question. 